\title{
Numerical study of sub-millimeter Gunn oscillations in InP and GaN vertical diodes: dependence on DC bias, doping and length
}

\author{
S. García, I. Íñiguez-de-la-Torre, S. Pérez, J. Mateos and T. González \\ Departamento de Física Aplicada, Universidad de Salamanca, \\ Plaza de la Merced s/n, 37008 Salamanca, Spain
}

\begin{abstract}
In this work, we report on Monte Carlo simulations of InP and GaN vertical Gunn diodes to optimize their oscillation frequency and DC to AC conversion efficiency. We show that equivalent operating conditions are achieved by the direct application of a sinusoidal AC voltage superimposed to the $\mathrm{DC}$ bias and by the simulation of the intrinsic device coupled with the consistent solution of a parallel RLC resonant circuit connected in series. InP diodes with active region about $1 \mu \mathrm{m}$ offer a conversion efficiency up to $5.5 \%$ for frequencies around $225 \mathrm{GHz}$. By virtue of the larger saturation velocity, for a given diode length, oscillation frequencies in $\mathrm{GaN}$ diodes are higher than for InP structures. Current oscillations at frequencies as high as $675 \mathrm{GHz}$, with $0.1 \%$ efficiency, are predicted at the sixth generation band in a $0.9 \mu \mathrm{m}-$ long $\mathrm{GaN}$ diode, corroborating the suitability of $\mathrm{GaN}$ to operate near the $\mathrm{THz}$ band. At the first generation band, structures with notch in general provide lower oscillation frequencies and efficiencies in comparison with the same structures without notch. However, a higher number of generation bands are originated in notched diodes, thus typically reaching larger frequencies. Self-heating effects reduce the performance, but in GaN diodes the efficiency is not significantly degraded.
\end{abstract}




\section{INTRODUCTION}

The electromagnetic waves within the frequency range $100 \mathrm{GHz}-10 \mathrm{THz}$, which are situated between the microwaves and the infrared light in the electromagnetic spectrum [1], are typically called Terahertz $(\mathrm{THz})$ waves. This band is attracting a big interest in the scientific community over the last years due to its multiple potential applications, and nowadays it exists a huge activity with the main objective of obtaining sources, amplifiers and detectors at those sub-millimeter frequencies. The generation in the $\mathrm{THz}$ range is not an easy task, and two approaches are possible, one coming from the electronics field and the other from optics [2]. Since the discovery of Gunn effect in the 60's [3-5], GaAs and InP Gunn diodes are classically used to generate signals up to a few hundreds of $\mathrm{GHz}(\sim 200 \mathrm{GHz}[6-8])$, but the intrinsic characteristics of these materials make it impossible to reach the $\mathrm{THz}$ range. One of the limitations of these materials comes from their saturation velocity $\left(v_{s}=0.91 \times 10^{7} \mathrm{~cm} / \mathrm{s}\right.$ for GaAs and $v_{s}=1.16 \times 10^{7} \mathrm{~cm} / \mathrm{s}$ for $\mathrm{InP}$ ), which is not very high, this parameter being crucial to reach oscillation frequencies close to the $\mathrm{THz}$ range. In addition, these materials have a low threshold and breakdown electrics fields [9], so that the application of high bias is not possible and thus the delivered power level is small. The solution may come by means of the use of $\mathrm{GaN}$, with higher threshold field and saturation velocity. Technological efforts to fabricate GaN diodes able to support the high bias voltage required to operate under negative differential resistance conditions will be necessary to successfully achieve high power oscillations in the range 200-400 GHz according to the predictions of various works [10], [11]. Indeed, experimental evidence of oscillations has not been achieved so far in GaN vertical diodes, mostly because of the proximity of the metal contacts and the very high applied voltages required for operation beyond the threshold field, which typically cause the diodes to burn out before oscillating [12], [13]. As alternative to InP and $\mathrm{GaN}$ vertical diodes, planar structures such as InGaAs/InAlAs [14], GaAs/AlGaAs[15], [16], [17] and InGaAs/AlGaAs planar diodes [18] and GaN/AlGaN self-switching-diodes (SSDs) [19] are currently being investigated.

Even though there are several works where vertical structures have been analyzed [20], there is not a systematic study where the performances of vertical diodes with different materials, lengths, bias conditions and doping levels are compared. The main objective of this work is to make a detailed study using a home-made Monte Carlo (MC) 
simulator [21]. The performance of $\mathrm{InP}$ and $\mathrm{GaN}$ diodes for different bias conditions, with and without notch, with lengths of the active region between $750 \mathrm{~nm}$ and $1500 \mathrm{~nm}$ and two doping schemes will be studied in terms of their oscillation frequencies and DC to $\mathrm{AC}$ conversion efficiency. In a real environment these vertical structures must operate embedded in a resonant circuit, typically a parallel RLC circuit connected in series with the diode [22]. In this paper we also demonstrate that the study of the diodes under a simple single-tone excitation is equivalent, in some cases, when appropriate circuit parameters can be found, to a self-consistent coupling of the MC code for the intrinsic device with the solution of the equations for the external resonant RLC circuit. The paper is organized as follows. In Sec. II, the details of the device structure, semiconductor materials and physical model used in the numerical simulations are described. In Sec. III, first the equivalence between a RLC circuit and a single-tone excitation is shown. Then, we present the results of the MC simulations of diodes with several lengths and two doping schemes, with and without notch, and discuss the dependence on the bias conditions. Finally, Sec. IV summarizes the main conclusions.

\section{DEVICE STRUCTURE AND PHYSICAL MODEL FOR THE SIMULATION}

\section{II.1. Device structure}

A schematic representation of the studied devices is shown in Fig. 1. In this work we focus on the analysis of two types of diodes, with $\left(n^{+} n^{-} n n^{+}\right)$and without $\left(n^{+} n n^{+}\right)$notch. Note that for the diode with notch the active region $(L)$ is composed of a doped region $(n)$ and an initial lightly doped notch $\left(n^{-}\right)$. In both cases the active region is sandwiched between two heavily doped ohmic contact $n^{+}$regions, whose lengths are $100 \mathrm{~nm}$ and $300 \mathrm{~nm}$, respectively. The considered asymmetry is due to the fact that while the cathode is always at equilibrium, for high applied bias the hot electrons in the upper valleys reaching the anode require enough length to thermalize. Systematic simulations with the MC tool for different lengths of the active region, doping levels and two materials ( $\mathrm{InP}$ and $\mathrm{GaN}$ ) will be presented. The doping of the $n^{+}$regions is always $n^{+}=2 \times 10^{18} \mathrm{~cm}^{-3}$. We have simulated four different lengths, $L=1500,1200,900$ and 750 $\mathrm{nm}$. In the case of the structures with notch the length of the notch (always $250 \mathrm{~nm}$ ) is included in $L$. Regarding the doping levels we have considered two doping schemes denoted as DS1 and DS2. For DS1 we use $n=1 \times 10^{17} \mathrm{~cm}^{-3}$ and $n^{-}=2 \times 10^{16} \mathrm{~cm}^{-3}$ while for DS2 $n=5 \times 10^{17} \mathrm{~cm}^{-3}$ and $n^{-}=1 \times 10^{17} \mathrm{~cm}^{-3}$. It is to be noted that a ratio of $n / n^{-}=5$ is kept. 


\section{II.2. Material properties and physical model for the simulations}

For the intrinsic study of the devices under analysis, a semi-classical ensemble MC simulator self-consistently coupled with a 2D Poisson solver [21], successfully tested is several devices, is employed [23], [24]. The conduction band of InP and GaN is modeled by three non-parabolic spherical valleys. InP and $\mathrm{GaN}$ are direct band-gap semiconductors and, furthermore, $\mathrm{GaN}$ is a wideband semiconductor. Under normal conditions, InP crystallizes in the Zinc-Blende structure and in our model $\Gamma, \mathrm{L}$ and $\mathrm{X}$ valleys are considered [25]. GaN can crystallize in three types of structures (Wurzite, Zinc-Blende and Rocksalt) [26]. In this work, a Wurzite crystal structure will be consider, modeled by $\Gamma_{1}, U$ and $\Gamma_{3}$ valleys where the lowest satellite valley sitting in the so-called $\mathrm{U}$ point is $2.2 \mathrm{eV}$ above the conduction band minimum at $\Gamma_{1}$ [25]. All mayor scattering mechanisms, such as intervalley phonons, acoustic, polar modes (optical and non-optical) and ionized impurities, are considered for InP based diodes. In addition for GaN structures the piezoelectric scattering is also taken into account. The most relevant properties at room temperature for the two semiconductors materials are summarized in Table 1 [25]. As the saturation velocity is higher in $\mathrm{GaN}, \mathrm{GaN}$ diodes are expected to operate at higher frequencies. Additionally this material has a larger GAP and separation between the satellite and central valleys in the conduction band, thus exhibiting higher threshold and breakdown electric fields and allowing applying higher bias before the breakdown, which is the main advantage of using GaN in order to achieve higher AC power emission.

\begin{tabular}{|c|c|c|}
\cline { 2 - 3 } \multicolumn{1}{c|}{ GAP $(\mathrm{eV})$} & InP & GaN \\
\hline Electron mobility $\left(\mathbf{c m}^{\mathbf{2}} \mathbf{\mathrm { Vs }}\right)$ & 1.34 & 3.44 \\
\hline Peak velocity $\left(\mathbf{1 0 ^ { 7 }} \mathbf{c m} / \mathbf{s}\right)$ & 2.30 & 2.53 \\
\hline Saturation velocity $\left(\mathbf{1 0 ^ { 7 }} \mathbf{c m} / \mathbf{s}\right)$ & 1.16 & 1.43 \\
\hline Threshold electric field $(\mathbf{K V} / \mathbf{c m})$ & 13.0 & 210.0 \\
\hline Breakdown electric field $(\mathbf{M V} / \mathbf{c m})$ & 0.5 & 3.3 \\
\hline
\end{tabular}

Table 1: Relevant properties of $\mathrm{InP}$ and $\mathrm{GaN}$ at room temperature. 
In order to analyze the diodes' capability as emitters, the dissipated DC power, $P_{D C}$, and the time-average AC power, $P_{A C}$, are evaluated, and the conversion efficiency, $\eta$, is calculated as the ratio between $P_{A C}$, and $P_{D C}$, with a minus sign

$$
\eta=-\frac{P_{A C}}{P_{D C}}
$$

In this context, negative values of $\eta$ indicate a resistive behavior of the diode, while positive values mean AC generation from DC. A common technique to determine $\eta$ is the direct application of a signal $V_{d}(t)$ between the diode terminals consisting in a single-tone sinusoidal potential of amplitude $V_{A C}$ superimposed to the DC bias $V_{D C}[19]$ :

$$
V_{d}(t)=V_{D C}+V_{A C} \cos (2 \pi f t) .
$$

We will compare the value of the signal applied between the diode terminals $V_{d}(t)$ calculated from Eq. (2) with the one obtained when only the DC bias is applied but the diode is connected in series with a parallel RLC resonant circuit [see Fig. 2]. The equations of such circuit can be written as follows [22]

$$
V_{D C}=V_{d}+V_{C} \text {, }
$$

where $V_{d}$ and $V_{C}$ are the voltage drops at the terminals of the diode and the RLC circuit, respectively. We need to solve the Poisson's equation together with the external circuit equations. The total current density flowing through the diode is

$$
j_{d}=\mathrm{C} \frac{d V_{\mathrm{C}}}{d t}+j_{\mathrm{L}}+\frac{V_{\mathrm{C}}}{\mathrm{R}},
$$

with $j_{L}$ the current density flowing through the inductance L.

$$
V_{\mathrm{C}}=\mathrm{L} \frac{d j_{\mathrm{L}}}{d t} .
$$

According to the generalized Ramo-Shockley Theorem [27], the current flowing through the diode has two contributions

$$
j_{d}=j_{v}+\mathrm{C}_{\mathrm{d}} \frac{d V_{d}}{d t}
$$

where $j_{v}$ is the current caused by the motion of the charged particles, and the second term is the displacement current due to the time-varying potentials at the electrodes, with $C_{d}=\varepsilon_{0} \varepsilon_{r} / l$ the diode geometrical capacitance (with $\varepsilon_{0}$ the permittivity of free space, $\varepsilon_{r}$ the static relative dielectric constant and $l$ the total diode length).

By means of a finite differences technique and considering a time-independent source signal, one obtains 


$$
V_{d}(t)=\frac{-j_{v}(t)+\frac{\mathrm{C}_{\mathrm{d}}}{\Delta t} V_{d}(t-\Delta t)+\frac{V_{D C}}{\mathrm{R}}+j_{L}(t-\Delta t)+\frac{\Delta t}{\mathrm{~L}} V_{D C}+\frac{\mathrm{C}}{\Delta t} V_{d}(t-\Delta t)}{\frac{\mathrm{C}_{\mathrm{d}}}{\Delta t}+\frac{1}{\mathrm{R}}+\frac{\Delta t}{\mathrm{~L}}+\frac{\mathrm{C}}{\Delta t}} .
$$

$V_{d}(t)$ is updated at each time step $(\Delta t)$ by using this expression before solving the Poisson's equation in the device [22], [28]. In next section we demonstrate that in some cases the excitation obtained from Eq. (7) is equivalent, by a proper selection of the R, $\mathrm{L}$ and $\mathrm{C}$ parameters, to the operation of the diode under a single tone excitation like that of Eq. (2).

Around 300000 iterations with a time-step of 1 fs are considered in our simulations. The number of electrons simulated is in the range 25000-50000 and all the results presented in this article are at room temperature.

\section{RESULTS AND DISCUSSION}

\section{III.1. Equivalence of RLC circuit and single-tone excitation}

In Figure 3 the direct equivalence between the parallel RLC circuit and the single-tone excitation is shown. Here an InP diode without notch $\left(n^{+} n n^{+}\right)$, with DS1 and $L=900 \mathrm{~nm}$ is considered. In Fig. 3(a) we plot the results for a simulation using $V_{D C}=7.5 \mathrm{~V}$ and $\mathrm{R}=3 \times 10^{-9} \Omega \mathrm{m}^{2}, \mathrm{~L}=4.7 \times 10^{-23} \mathrm{Hm}^{2}$, and $\mathrm{C}=0.0081 \mathrm{~F} / \mathrm{m}^{2}$, leading to a quality factor $Q \sim 40$. In Fig. 3(b) a single-tone scheme of $243 \mathrm{GHz}$ and $V_{A C}=1.9 \mathrm{~V}$, with $V_{D C}=7.5 \mathrm{~V}$, is used. As clearly observed, both operating conditions are equivalent as long as the values considered for $R, L$ and $C$ in the resonant circuit lead to a quasi-sinusoidal signal of the same amplitude as that of the single-tone excitation [Fig. 3(b)]. A phase shift slightly bigger than $\pi / 2$ between the current density and the applied voltage appears, which means that the diode exhibits a positive efficiency $\eta$ in such conditions. In the case of the use of the resonant RLC circuit the mean current density is $I_{m}=39 \times 10^{8} \mathrm{~A} / \mathrm{m}^{2}$ and $\eta=1.9 \%$, while applying the single-tone we obtain, $I_{m}=38.2 \times 10^{8} \mathrm{~A} / \mathrm{m}^{2}$ and $\eta=2.0 \%$. Consequently, and for this particular case, both methods are essentially equivalent. In the subsequent analysis, in order to be systematic, and at the expense of providing results that in some cases could correspond to non-realistic conditions, we will make use of the single-tone excitation [Eq. (2)] because it is less time-consuming than the resonant-circuit simulation.

Indeed, in the above case the $Q$ factor of the parallel RLC resonant circuit is big enough to filter the rest of possible harmonics and thus provide a pure sinusoidal excitation. 
However, in other cases it was not possible to replicate a pure sinusoidal excitation by using the simple parallel RLC circuit considered here. It would require including a further band-pass filter, but circuit design goes beyond the objective of this paper.

Thus, the results obtained under the single-tone excitation illustrate the intrinsic potentiality of the diodes to generate signals at different frequency bands, but are obtained under optimum conditions, in some cases not straightforwardly affordable experimentally.

\section{III.2. Results for DS1}

\section{III.2.a. Static IV curves}

As a first step, the dependence of the current density on the bias for diodes, with and without notch, with different lengths of the active region and both for $\mathrm{InP}$ and $\mathrm{GaN}$ is shown in Fig. 4. The saturation of the current takes place with the onset of intervalley transfer mechanisms. The shorter the device the higher the internal electric field for a given bias, and thus the smaller the bias required for saturation. In the longest InP-based diodes without notch $(1500 \mathrm{~nm}$ and $1200 \mathrm{~nm})$, it is noteworthy the presence of an abrupt decrease in the average current density for bias voltages of around $5 \mathrm{~V}$. The origin of this change is attributed to a variation in the oscillation regime from accumulation-layer mode to transit-time dipole-layer mode [29]. It is to be noted that for $\mathrm{GaN}$ the applied bias is higher than for InP because intervalley transfer and negative differential mobility in GaN appears at much higher electric fields. Finally, while in InP based diodes the notch is limiting the current density, which in saturation is essentially the same for all the lengths, such is not the case in GaN. This is an indication that this notch in the GaN diodes is not being effective and presumably it will not fix the position of the domain onset. This will be studied in detail in next sections.

\section{III.2.b. DC to AC conversion efficiency, effect of the notch in InP}

In order to analyze the performance of the diodes operating as emitters, the DC to AC conversion efficiency, $\eta$, has been evaluated by means of the superposition of a singletone sinusoidal potential of amplitude $V_{\mathrm{AC}}$ to a DC bias $V_{\mathrm{DC}}$. The dependence of $\eta$ on the frequency $f$ of the $\mathrm{AC}$ excitation for an InP-based diode with $L=900 \mathrm{~nm}$ (DS1) and with $V_{D C}=12.0 \mathrm{~V}$ and $V_{A C}=1.5 \mathrm{~V}$ is shown in Fig. 5. Higher frequencies are achieved in the diode without notch in comparison with the diode with notch for the first generation 
band. This is basically because the notch fixes the onset of the charge accumulation domain in the cathode region while in the diode without notch it takes place well inside the active region.

In the case of the diode with notch three bands with $\eta>0$, indicating generation of AC power, are observed. The frequency at which the maximum efficiency is achieved (frequency of maximum efficiency, FME) for the first generation band is $\mathrm{FME}_{1}=137 \mathrm{GHz}$ with efficiency of $0.92 \%$, for the second generation band is $\mathrm{FME}_{2}=238 \mathrm{GHz}$ with still a competitive efficiency of $\eta=0.28 \%$ and the third one at $\mathrm{FME}_{3}=369 \mathrm{GHz}$ with efficiency of $0.03 \%$. The bands potentially suitable for oscillations match with the range of frequencies where the real part of the impedance is negative $[\operatorname{Re}(Z)<0]$, as can be observed in the inset of Fig. 5 [30]. The exact values for the diode with notch are 71-156 GHz, 206-275 GHz and 357-390 GHz, where we have identified the presence of one, two or three charge accumulation domains inside the diode's active region. For the diode without notch we identify only two ranges where $\operatorname{Re}(Z)<0$ to be $104-237 \mathrm{GHz}$ and $335-364 \mathrm{GHz}$. A value of $1.84 \%$ for the efficiency is achieved for the first generation band at $206 \mathrm{GHz}$. It is remarkable that in all cases, as was found in Refs. [7], [8], the top limit (zero crossing) of the first negative band of the real part of the impedance approaches the self-oscillation frequency of the diode (without AC bias component).

\section{III.2.c. Dependence of the efficiency on the length, bias conditions and material}

Once we have explored the effect of the notch for InP, in this section we perform a systematic study of the frequency of the oscillations and the conversion efficiency, $\eta$. We analyze and compare their dependence on the length and the DC bias for both semiconductors, InP and GaN, using DS1.

Fig. 6 shows the values of FMEs in the different generation bands as a function of the diode length (studying until the third generation band, if it exists). The size of the bubbles scales with the DC to AC conversion efficiency, $\eta$, at such FMEs, whose value is also indicated. In the case of InP, for $V_{D C}=12.0 \mathrm{~V}$ and $V_{A C}=1.5 \mathrm{~V}$, the FMEs lie in the range of 70-400 GHz [Fig. (a)]. As explained before, the absence of notch provides better performance in terms of the values of $\eta$ and FME for the first generation band. In all cases the shorter the active region the higher the FME, but the efficiency is nearly constant. It is remarkable that in diodes without notch for $L=900 \mathrm{~nm}$ and $1500 \mathrm{~nm}$ the 
value of $\eta$ at the first generation band is similar $(1.84 \%)$, but the FME of the oscillations is $68.75 \mathrm{GHz}$ higher for the shorter one.

For GaN, Fig. 6(b), we use $V_{D C}=70 \mathrm{~V}$ and $V_{A C}=10 \mathrm{~V}$, and we obtain FMEs between 100-600 GHz. In this case, in contrast with InP, there is no significant difference between the results in presence and absence of notch. This indicates that the notch is not being effective at fixing the nucleation of the high field domain, and the onset of charge accumulation takes place at the same position inside the active region irrespectively of the presence or absence of notch. In GaN, electrons have higher effective mass than in InP, and they move more slowly and suffer many scattering mechanisms inside the notch. So, for the $n^{-} n$ gradient of DS1, and even if the electric field is quite high in the notch, they do not gain enough energy so as to promote to the upper U-valleys. The highest efficiency, $1.47 \%$, is achieved with a length of $L=1500 \mathrm{~nm}$ but for a small FME of $143 \mathrm{GHz}$, while the length providing the highest FME of $568 \mathrm{GHz}$ (in the third generation band) is $L=900 \mathrm{~nm}$, but the efficiency is very low, $0.09 \%$. Finally, the diode with notch and $L=1200 \mathrm{~nm}$ presents even a fourth generation band with a FME of 638 $\mathrm{GHz}$ but with very low efficiency of $0.04 \%$ (not included in this Figure, case that will be studied later).

Regarding the effect of the bias conditions on the frequency of the oscillations, Fig. 6(c) presents the results for $L=900 \mathrm{~nm}$ in $\mathrm{InP}$ and $\mathrm{GaN}$ diodes up to the second generation band. Let's start with the InP case, where $V_{A C}=1.5 \mathrm{~V}$ and $V_{D C}$ is swept from $3.5 \mathrm{~V}$ up to $12 \mathrm{~V}$. The range of FMEs found is $100-320 \mathrm{GHz}$. Diodes without notch present only one generation band in the range 206-243 GHz, while diodes with notch exhibit more than one in almost all the situations. The most relevant result is that the DC to AC conversion efficiencies achieved without notch are larger, exceeding $5 \%$, than those obtained with notch. For diodes without notch, as a general feature the higher the $V_{D C}$ value the lower the efficiency and the FME as a consequence of the higher electric field inside the structure (with the only exception of $V_{D C}=3.5 \mathrm{~V}$, voltage at which the oscillations are near to disappear and the FME decreases). However, when the diode has a notch, it fixes the oscillation frequency, and the FME shows practically no dependence on $V_{D C}$ for the first generation band, while the efficiency decreases for higher $V_{D C}$ as in the previous case. Again for $V_{D C}=3.5 \mathrm{~V}$ a different behaviour is found and only one generation band exists. The maximum efficiency for the first generation band is $2.22 \%$, corresponding to a FME of $150 \mathrm{GHz}$ and a bias $V_{D C}=6 \mathrm{~V}$. For this bias 
it is also observed that $\eta$ in the second generation band takes the highest value, $0.57 \%$ at $288 \mathrm{GHz}$.

In the case of GaN, we consider $V_{A C}=10 \mathrm{~V}$ and $V_{D C}$ is swept from $25 \mathrm{~V}$ up to $70 \mathrm{~V}$, values notably higher than in InP diodes. In this case the range of FMEs achieved is much larger, between 100 and $500 \mathrm{GHz}$. Again the presence of the notch seems to be not effective as there is no significant difference between the results of structures with and without notch. This means that the domain is always created inside the active region (at similar distance from the anode) and not in the vicinity of the cathode. As a consequence in the two cases for the first generation band $\eta$ and the FME decrease with the increase of $V_{D C}$, with FME in the range 200-243 GHz. For $V_{D C}<35 \mathrm{~V}$ the efficiency begins to decrease until the oscillation disappears completely as electrons do no reach enough energy to be in the negative differential mobility region. Even if the FMEs are the same, the diodes with notch provide higher efficiency in all cases except for $V_{D C}=70 \mathrm{~V}$ in the first generation band. It is expected that increasing the doping levels will improve the role played by the notch, what is studied in next section.

To conclude the study of the DS1, in Fig. 7 we show the efficiency $v s$. frequency for GaN-based diodes with $L=1200 \mathrm{~nm}\left(V_{D C}=70 \mathrm{~V}\right.$ and $\left.V_{A C}=10 \mathrm{~V}\right)$. In this particular case, as mentioned above, a fourth generation band at $638 \mathrm{GHz}$ appears in the notched diode. In the inset instantaneous profiles of carrier concentration along the diode with notch are plotted for different frequencies of the $\mathrm{AC}$ excitation $\mathrm{FME}=175,319,475$ and $638 \mathrm{GHz}$ (FMEs of each generation band). As expected, an increasing number (from 1 to 4) of charge domains drifting along the active region is observed for increasing frequencies of the AC excitation. The generation bands and the associated FMEs are not equally separated as the nucleation of several domains reduces the so-called dead space.

\section{III.3. Results for the DS2}

In order to enhance the effect of the notch in the GaN diodes, here we consider a second doping scheme, denoted as DS2, where the doping of the $n^{-}$and $n$ regions is larger. The results are summarized in Fig. 8. Firstly, in Fig. 8(a), where the $I-V$ curves in diodes (with and without notch) with different lengths are plotted, two main differences with respect to the case of DS1 [Fig. 4(b)] can be observed: i) diodes without notch present a more visible negative incremental resistance zone and ii) when present, the notch limits the current in the diodes, as happened in the InP diodes with DS1. This indicates that 
with the doping values of DS2 the notch is more effective. To confirm this expectation, in Fig. 8(b) we analyse again the dependence of the FME and maximum efficiency on the length. Now, there are noticeable differences between both types of diodes, with and without notch, being more prominent in the longer structures $(L=1200$ and $1500 \mathrm{~nm})$. With DS2, although the ratio in the concentration between the $n$ and $n^{-}$regions is the same $\left(n / n^{-}=5\right)$, the gradient is higher, thus increasing the electric field in the notch beyond the threshold value $\left(E_{T}=210 \mathrm{kV} / \mathrm{cm}\right)$, so that the presence of the notch is actually effective at fixing the point of formation of the domain. For the device with notch, up to a third generation band is visible, reaching $500 \mathrm{GHz}$ with significant efficiency. Furthermore, for $L=900 \mathrm{~nm}$ even the sixth generation band has been identified (not presented in the graphic), with a FME of $675 \mathrm{GHz}$, and for $L=750 \mathrm{~nm}$ the fifth one reaching $688 \mathrm{GHz}$.

Fig. 8(c) shows the FME versus the DC bias $V_{D C}$ in the diode with $L=900 \mathrm{~nm}$ (with and without notch) when $V_{A C}=10 \mathrm{~V}$ and $V_{D C}$ is swept from $25 \mathrm{~V}$ up to $70 \mathrm{~V}$. Only one generation band is observed in the diode without notch, where the best $\eta$ is obtained for $35 \mathrm{~V}$. Indeed, higher $\mathrm{DC}$ to $\mathrm{AC}$ maximum conversion efficiencies and FMEs are obtained for decreasing bias down to $35 \mathrm{~V}$. However it is the structure with notch which provides, for a bias of $25 \mathrm{~V}$, the highest FME (312 GHz) but with less efficiency. It is remarkable that FMEs close to $500 \mathrm{GHz}$ in the third generation band can be achieved when $V_{D C}$ is $55 \mathrm{~V}$ with an efficiency of $0.23 \%$.

Finally, to study the effect of the amplitude of the AC excitation, $V_{A C}$, we calculate the values of $\eta$ for a GaN-based diode with notch and $L=900 \mathrm{~nm}$ for the DS2, Fig. 9. Three values of $V_{A C}=6.5 \mathrm{~V}, 8.5 \mathrm{~V}$ and $10 \mathrm{~V}$ have been considered, with $V_{D C}=70 \mathrm{~V}$. When the $V_{A C}$ component is reduced, a lower $\eta$ but at slightly higher FMEs is achieved for the first and second generation bands. The same behavior has been observed for the DS1 in InP and $\mathrm{GaN}$ diodes (results not presented in this paper). For higher generation bands, the FMEs do not vary significantly. On the other hand, self-heating effects can be substantial at the high power values managed in the diodes, and could deteriorate their performance. To check this extent, we have simulated the structure presented before when $V_{D C}=70 \mathrm{~V}$ and $V_{A C}=10 \mathrm{~V}$, but at $500 \mathrm{~K}$ [Fig. 9]. It can be observed that in comparison with the result at room temperature, the FMEs are smaller for the different bands and $\eta$ is slightly lower. This effect obviously takes place because of the smaller drift velocity of electrons at higher temperature. Anyway, a fifth generation band where 
the FME is $625 \mathrm{GHz}$ with an efficiency of $0.06 \%$, and a fourth one with $\eta=0.15 \%$ at $475 \mathrm{GHz}$, are still obtained. We hope that such excellent predictions for $\mathrm{GaN}$ diodes can be confirmed experimentally soon.

\section{CONCLUSIONS}

By means of MC simulations, the equivalence between the operation of a diode connected in series with a resonant circuit and the direct application of a signal consisting in the superposition of a sinusoidal AC component to the $\mathrm{DC}$ bias has been proved in a particular example case, in which the feedback signal obtained from the RLC circuit was essentially sinusoidal. The latter technique has been used to analyze the performance of InP and GaN structures. GaN has high threshold and breakdown electric fields and thus requires higher bias (one order of magnitude higher than InP) for achieving negative differential mobility, what implies the possibility of generating higher $\mathrm{AC}$ power at the expense of an also higher DC consumption.

For InP diodes we have proved that the presence of a notch localizes the nucleation of the charge accumulation domain near the cathode and not inside the active region as happens in the structures without notch. In these diodes, oscillating frequencies up to a few hundreds of $\mathrm{GHz}(<300 \mathrm{GHz})$ have been found with competitive efficiencies. For the same doping scheme (DS1), the notch is not effective in GaN diodes, because the electric field in the notch region is not large enough to promote electrons to the $U$ valley. For this reason, a second doping scheme with higher values (DS2) has been studied. When the DS2 is considered, the notch is more effective and the domain is formed close to the cathode. The generation bands in GaN diodes take place at higher frequencies than in InP, corroborating the expectations to attain emission near the socalled terahertz gap. The best result achieved is a FME of about $600 \mathrm{GHz}$ for diodes with $L \approx 1 \mu \mathrm{m}$ for the two doping schemes. The selection of a DC voltage biasing the diode just in the initial part of the negative differential mobility region leads to a higher DC to AC conversion efficiency. Also it is observed in the simulations that the value of $\eta$ increases with the amplitude of the single-tone AC excitation. Self-heating effects lead to a reduction in the FMEs and the generation of less harmonics, but the device is still operative at the expected and promising values.

By using a single tone excitation, the intrinsic potentiality of GaN Gunn diodes for emission at different high-frequency bands has been shown. However, in order to 
actually extract the predicted AC power, an appropriate RLC circuit and tuneable bandpass filters should be designed to accomplish such ideal conditions. Mismatch, losses, heating effects, diode parasitic capacitances, etc. may additionally degrade the performance foreseen by MC simulations.

\section{ACKNOWLEDGMENTS}

This work has been partially supported by the European Commission through the ROOTHz Project ITC-2009-243845, by the Dirección General de Investigación (MICINN) through the Project TEC2010-15413 and by the Consejería de Educación de la Junta de Castilla y León through the Project SA183A121.

\section{REFERENCES}

[1] G. L. Carr, M. C. Martin, W. R. McKinney, K. Jordan, G. R. Neil and G. P. Williams. Nature, 420, 153 (2002).

[2] M. Tonouchi. Nat. Photonics, 1, 97 (2007).

[3] J. B., Gunn. Solid State Commun., 1, 88 (1963).

[4] B. K. Ridley and T. B. Watkins. Proc. Phys. Soc., 78, 293 (1961).

[5] K. Kurokawa. The Bell System Technical Journal, 48, 1937 (1969).

[6] M. Abe, S. Yanagisawa, O. Wada and H. Takanashi. JPN. J. Appl. Phys., 14, 70 (1975).

[7] V. Gruzinskis, E. Starikov, P. Shiktorov, L. Reggiani, M. Saraniti, and L. Varani. Simulation of Semiconductor Devices and Processes, 5, 333 (1993).

[8] V. Gruzinskis, E. Starikov, P. Shiktorov, L. Reggiani, and L. Varani. J. Appl. Phys, 76, 5260 (1994).

[9] E. Alekseev and D. Pavlidis. Solid-State Electron., 44, 941 (2000).

[10] R. F. Macpherson, G. M. Dunn and N. J. Pilgrim. Semicond. Sci. Technol, 23, 055005 (2008).

[11] C. Sevik and C. Bulutay. Appl. Phys. Lett., 85, 3908 (2004).

[12] O. Yilmazoglu, K. Mutamba, D. Pavlidis and T. Karaduman. IEEE Trans. Electron Devices, 55, 1563 (2008). 
[13] O. Yilmazoglu, K. Mutamba, D. Pavlidis and T. Karaduman. Electronics Lett., 43, 480 (2007).

[14] S. Pérez, T. González, D. Pardo and J. Mateos. J. Appl. Phys, 9, 094516 (2008).

[15] A. Khalid, N. J. Pilgrim, G. M. Dunn, C. Holland, C. R. Stanley, I. G. Thayne and D. R. S. Cumming. IEEE Trans. Electron Devices, 28, 849 (2007).

[16] N. J. Pilgrim, A. Khalid, G. M. Dunn and D. R. S. Cumming. Semicond. Sci. Technol., 23, 075013 (2008).

[17] M. Montes, G. Dunn, A. Stephen, A. Khalid, C. Li, D. Cumming, C. H. Oxley, R. H. Hopper, and M. Kuball. IEEE Trans. Electron Devices, 59, 654 (2012).

[18] C. Li, A. Khalid, S. H. Paluchowski Caldwell, M. C. Holland, G. M. Dunn, I. G. Thayne, D. R. S. Cumming. Solid-State Electron., 64, 67 (2011).

[19] A. Íñiguez-de-la-Torre, I. Íñiguez-de-la-Torre, J.Mateos, T.González, P. Sangaré, M. Gaucher, B. Grimbert, V. Brandli, G. Ducournau and C. Gaquière. J. Appl. Phys., 111, 113705 (2012).

[20] L. Yang, S. Long, X. Guo and Y. Hao. J. Appl. Phys., 111, 104514 (2012).

[21] C. Jacoboni and P. Lugli, The Monte Carlo method for semiconductor device simulation (Springer-Verlag, New York, 1989).

[22] P. Shiktorov, E. Starikov, V. Gruzinskis, S. Pérez, T. González, L. Reggiani, L. Varani and J. C. Vaissière. Semicond. Sci. Technol., 21, 550 (2006).

[23] I. Iñiguez-de-la-Torre, T. González, H. Rodilla, B. G. Vasallo and J. Mateos, Monte Carlo Simulation of Room Temperature Ballistic Nanodevices. Applications of Monte Carlo Method in Science and Engineering, (Prof. Shaul Mordechai, Croatia, 2011).

[24] Rodilla H., González T., Moschetti G., Grahn J. and Mateos J. Semicond. Sci. Technol., 26, 025004 (2011).

[25] O. Madelung, Semiconductors: Data Handbook. $3^{\text {rd }}$ edition, (Springer-Verlag, New York, 2004).

[26] E. López and J. Arrigaga. Superficies y Vacío. 17 (1), 21 (2004).

[27] H. Kim, H. S. Min, T. W. Tang and Y. J. Park. Solid-State Electron., 34, 1251 (1991). 
[28] S. García, B. G. Vasallo, J. Mateos and T. González. $9^{\text {th }}$ Spanish Conference on Electronic Devices (CDE 2013), IEEE Catalog CFP 13589, 79 (2013).

[29] S. M. Sze, K. K. Ng., Physics of Semiconductor Devices. $3^{\text {rd }}$ edition, (Wiley \& Sons, Inc., Publication, New Jersey, 2007).

[30] G. M. Dunn and M. J. Kearney. Semicond. Sci. Technol. 18, 794 (2003). 


\section{FIGURES AND CAPTIONS}
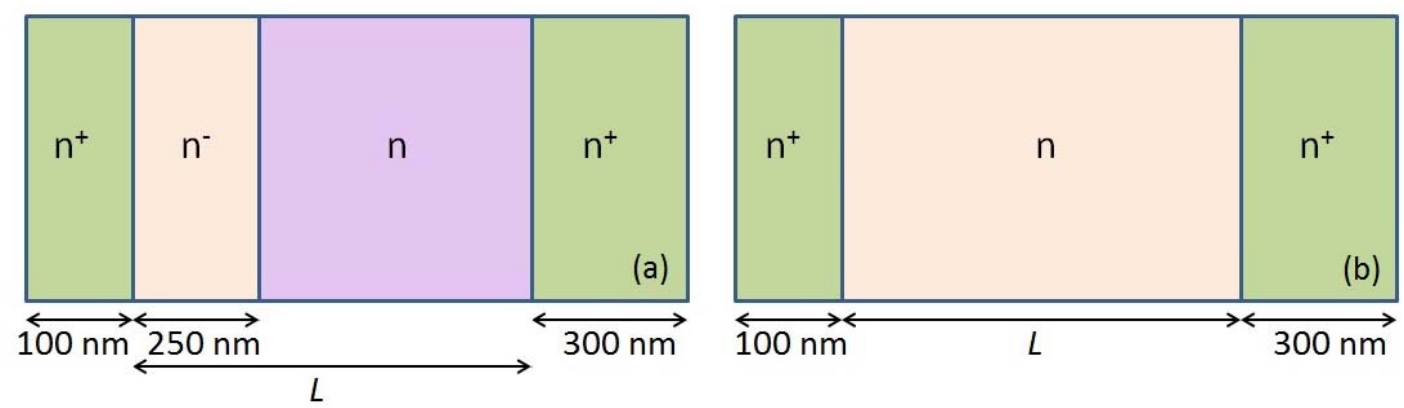

FIGURE 1. Geometry of the studied structures with physical dimensions. (a) Diode with a $250 \mathrm{~nm}$-length notch placed next to the cathode and (b) diode without notch. The lengths of $n^{+}$contact regions are $100 \mathrm{~nm}$ and $300 \mathrm{~nm}$ at the cathode and anode, respectively, in both structures. $n^{+}=2 \times 10^{18} \mathrm{~cm}^{-3}$, DS1: $n=1 \times 10^{17} \mathrm{~cm}^{-3}$ and $n^{-}=2 \times 10^{16} \mathrm{~cm}^{-3}$ and DS2: $n=5 \times 10^{17} \mathrm{~cm}^{-3}$ and $n^{-}=1 \times 10^{17} \mathrm{~cm}^{-3}$. Four different lengths, $L=1500,1200,900$ and $750 \mathrm{~nm}$ have been simulated. 


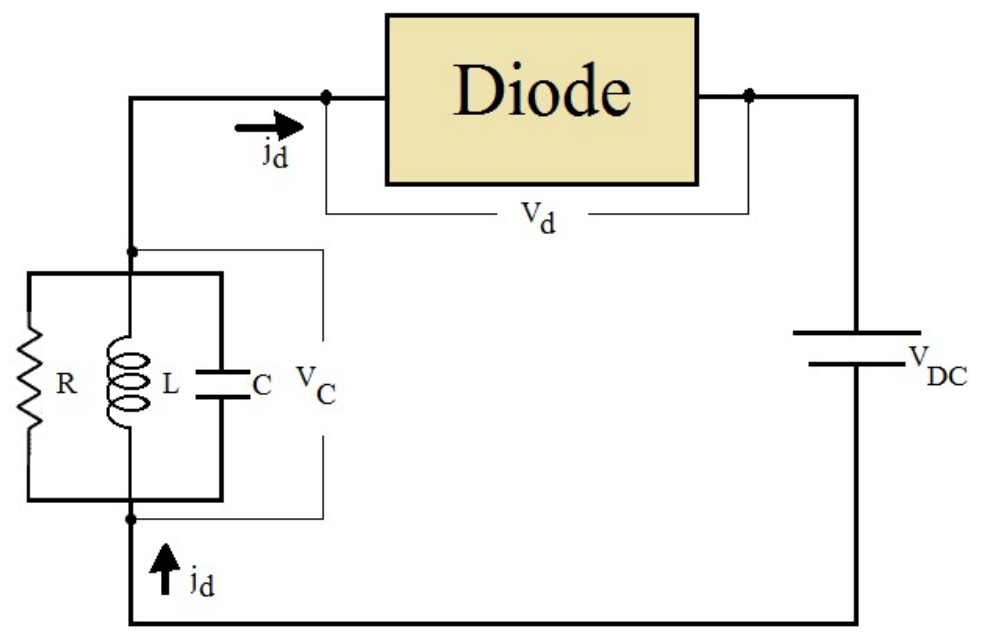

FIGURE 2. Scheme of the parallel RLC circuit connected in series with a diode. 


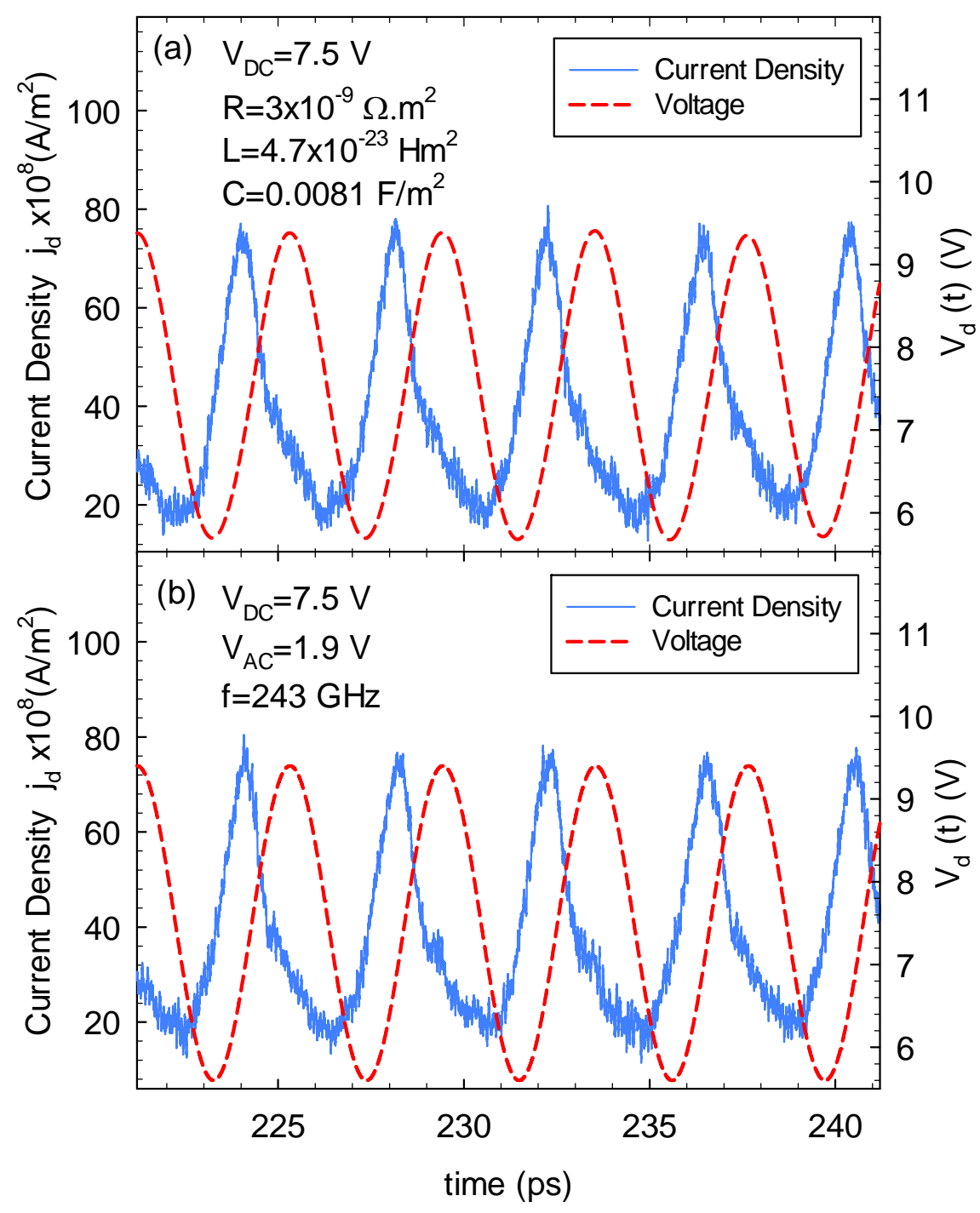

FIGURE 3. Time-sequences of current density $j_{d}$ and applied voltage $V_{d}(\mathrm{t})$ in a $\operatorname{InP} n^{+} n n^{+}$diode using the DS1 and $V_{D C}=7.5 \mathrm{~V}$. (a) Resonant RLC circuit in series with the diode $\left(R=3 \times 10^{-9} \Omega \mathrm{m}^{2}, L=4.7 \times 10^{-23} \mathrm{Hm}^{2}\right.$, and $C=0.0081 \mathrm{~F} / \mathrm{m}^{2}$ ) and (b) excitation by means of a single-tone of frequency $243 \mathrm{GHz}$ and $V_{A C}=1.9 \mathrm{~V}$ [Eq. 3]. 

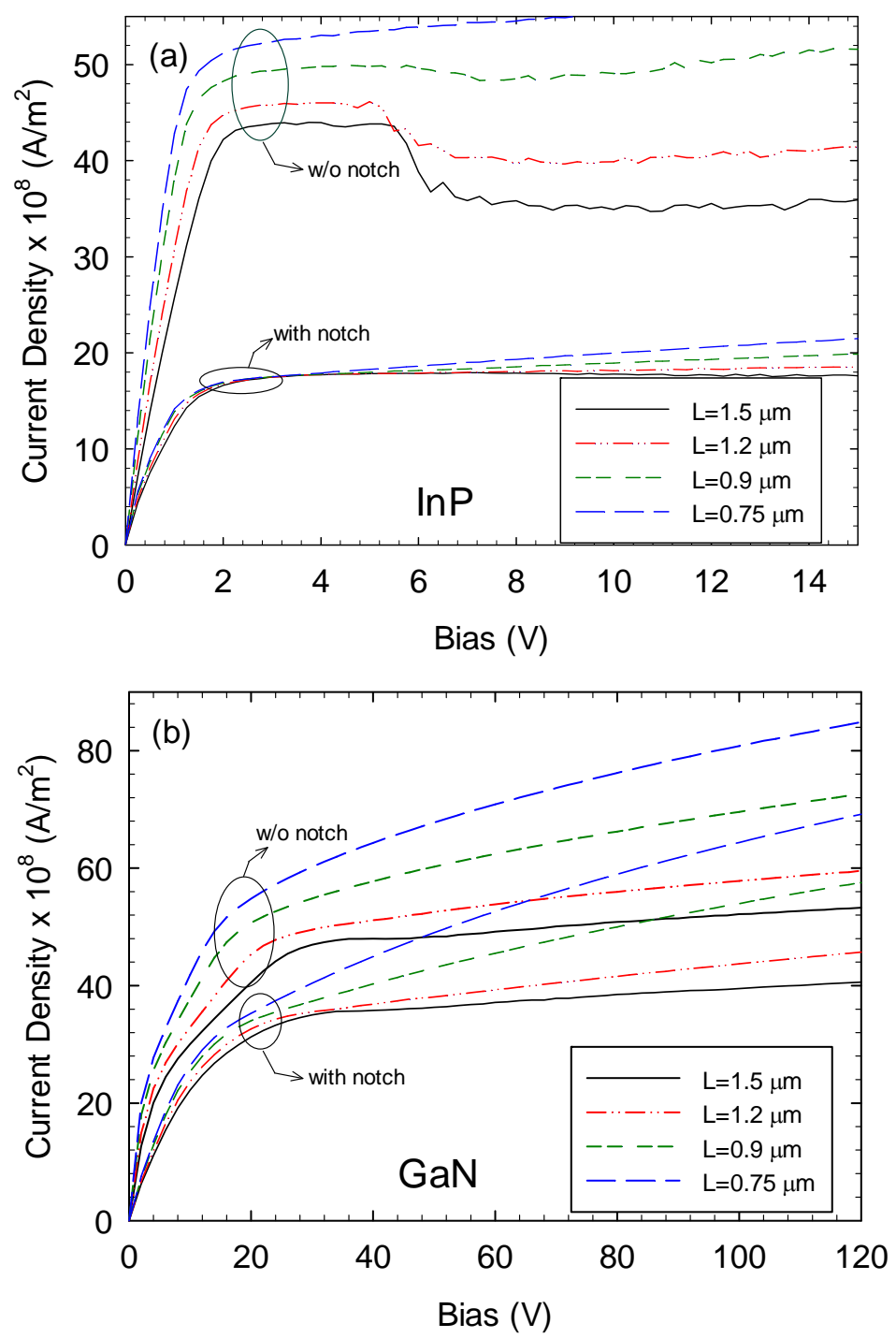

FIGURE 4. $I-V$ curves for the two types of diodes, with and without notch, and for different lengths of the active region: $L=1500 \mathrm{~nm}, 1200 \mathrm{~nm}, 900 \mathrm{~nm}$ and $750 \mathrm{~nm}$ for the DS1 and for (a) InP and (b) GaN. 


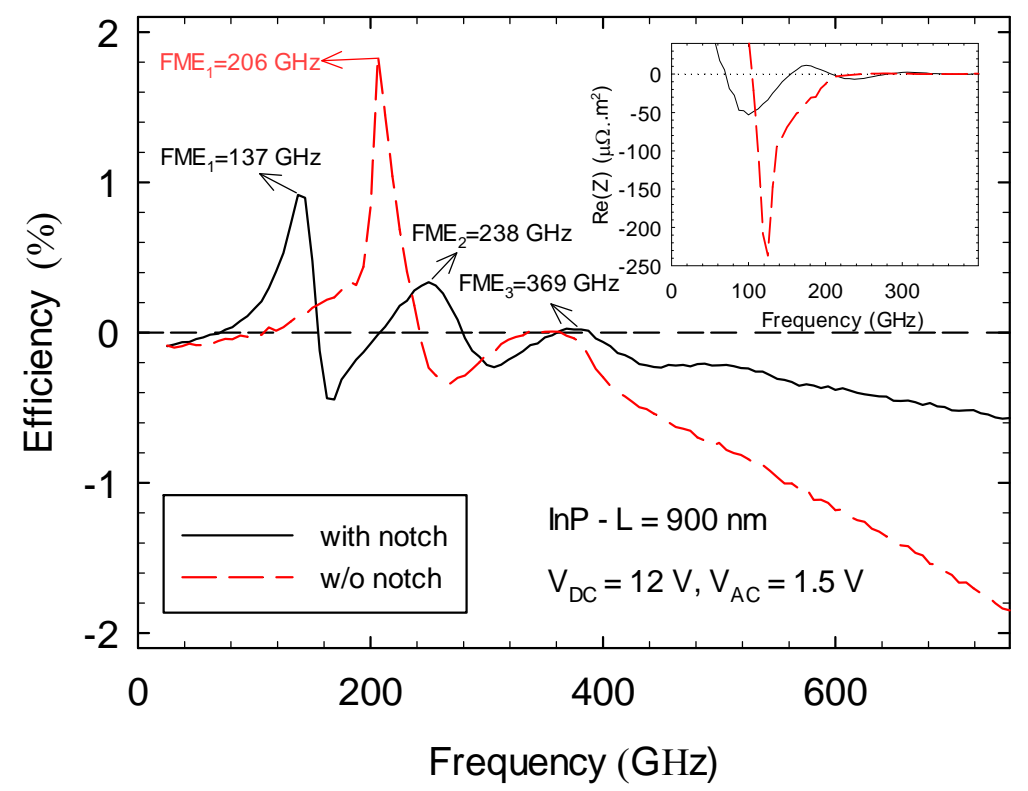

FIGURE 5. DC to AC conversion efficiency vs. frequency for InP-based diodes (with and without notch) with $L=900 \mathrm{~nm}$ and for the DS1. Single-tone excitation with $V_{D C}=12 \mathrm{~V}$ and $V_{A C}=1.5 \mathrm{~V}$. The inset shows the real part of the impedance. 

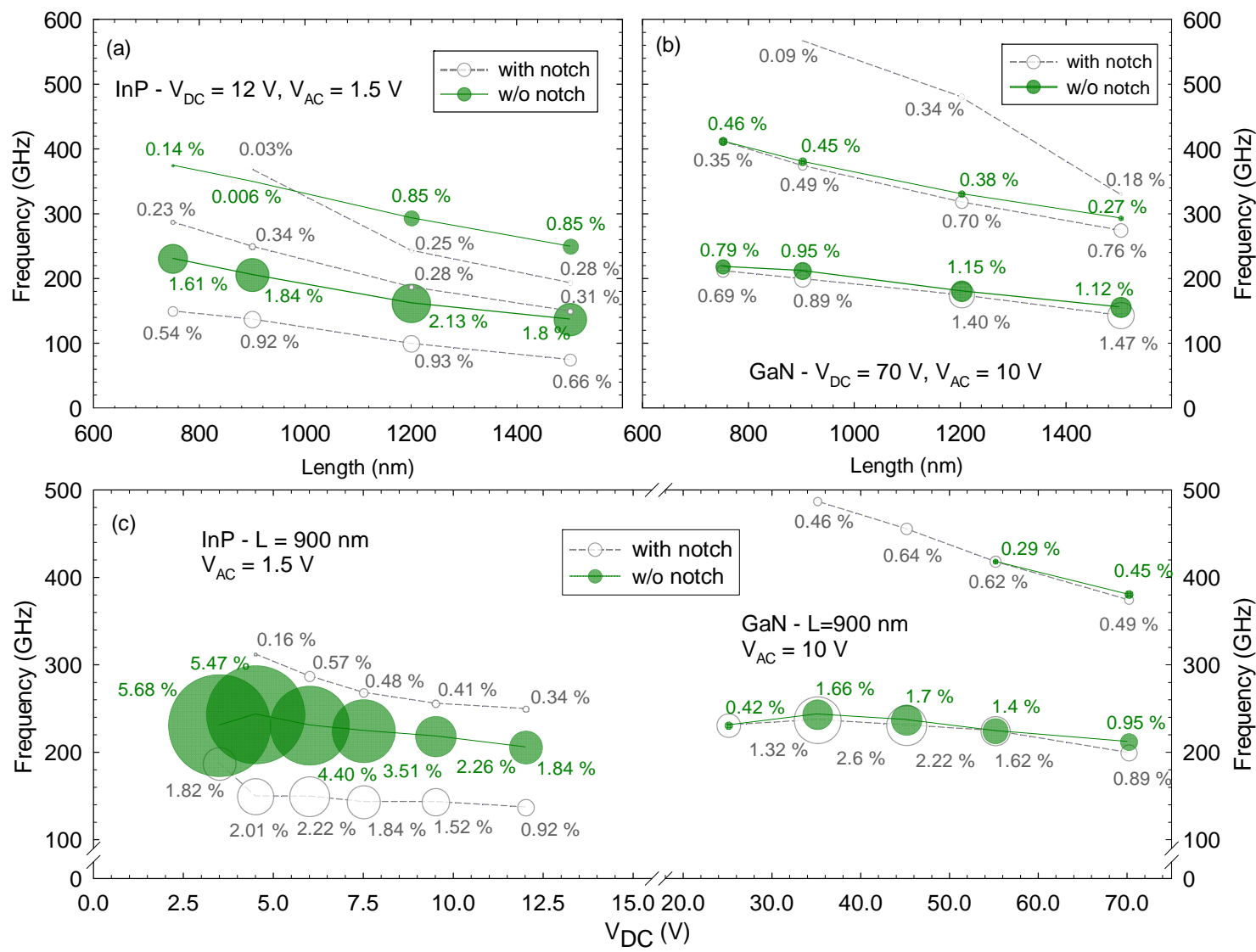

FIGURE 6. FME and corresponding efficiency of the different generation bands found in diodes with the DS1 with and without notch. The size of the bubbles scales with the maximum of the DC to AC conversion efficiency, $\eta$, indicated for the different generation bands. The dependence on the length of the active region is shown for (a) InP diodes using $V_{D C}=12 \mathrm{~V}$ and $V_{A C}=1.5 \mathrm{~V}$ and (b) GaN diodes using $V_{D C}=70 \mathrm{~V}$ and $V_{A C}=10 \mathrm{~V}$. (c) Dependence on the DC bias for $L=900 \mathrm{~nm}$ and using and AC excitation of $V_{A C}=1.5 \mathrm{~V}$ for $\mathrm{InP}$ and $V_{A C}=10 \mathrm{~V}$ for $\mathrm{GaN}$. 


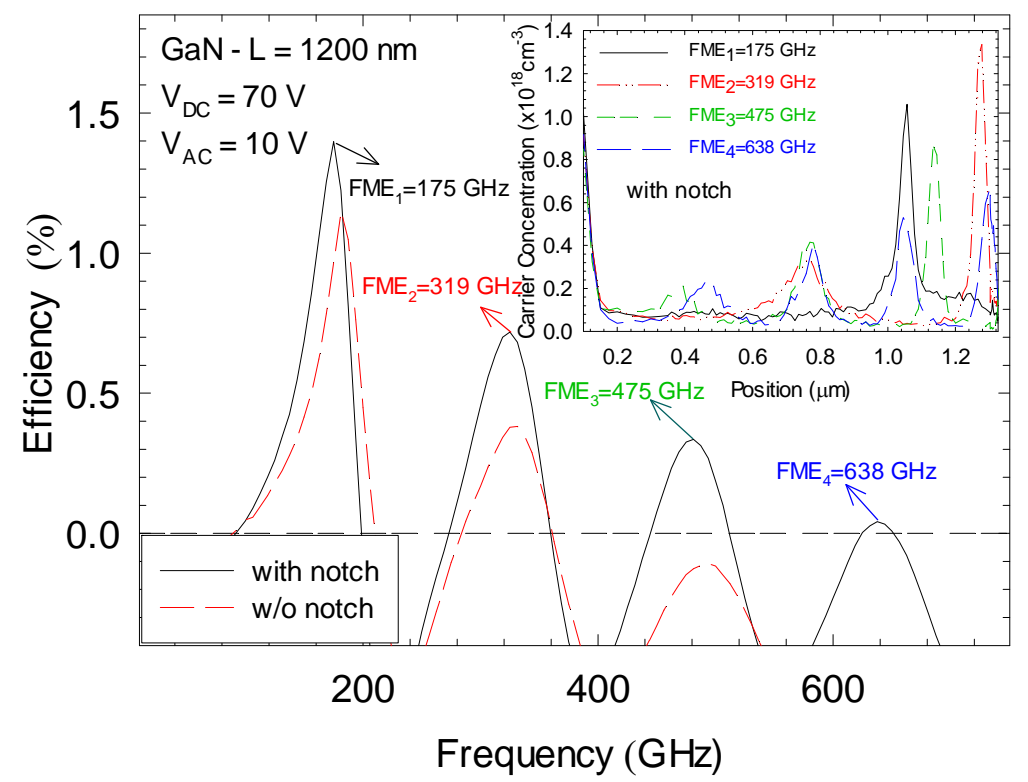

FIGURE 7. DC to AC conversion efficiency vs. frequency for GaN-based diodes (with and without notch) with $L=1200 \mathrm{~nm}$ and for the DS1. Single-tone excitation with $V_{D C}=70 \mathrm{~V}$ and $V_{A C}=10 \mathrm{~V}$. The inset shows the profile of carrier concentration along the diode corresponding to a given time within one period of the AC signal for frequencies at the maximum of the emission bands to be FME=175, 318.7, 475 and $637.5 \mathrm{GHz}$. 

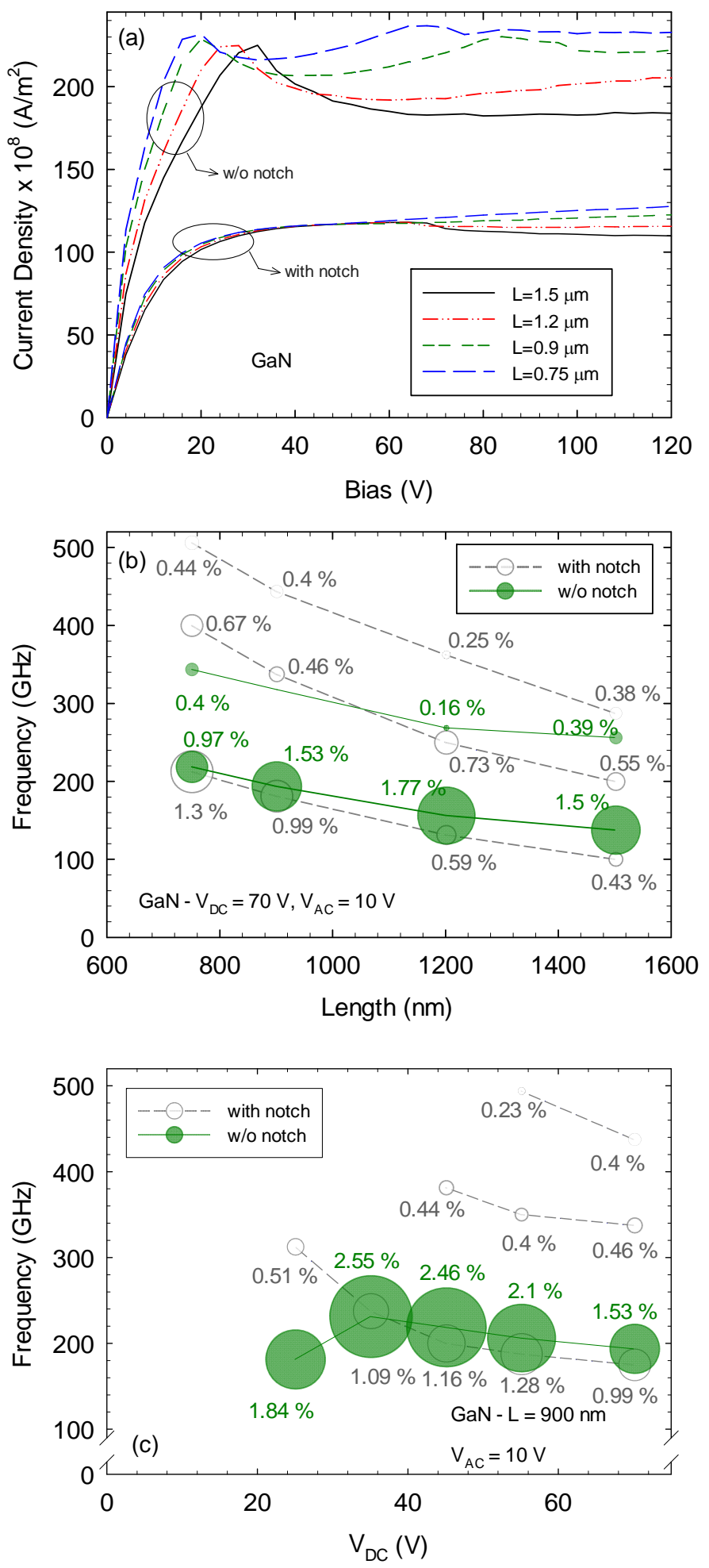

FIGURE 8. Results in a GaN diode (with and without notch) when the DS2 is considered. (a) $I-V$ curves for different lengths of the active region: $L=1500 \mathrm{~nm}, 1200 \mathrm{~nm}, 900 \mathrm{~nm}$ and $750 \mathrm{~nm}$. Dependence of the FME and corresponding efficiency of the different generation bands on the (b) length of the active region $\left(V_{D C}=70 \mathrm{~V}\right.$ and $V_{A C}=10 \mathrm{~V}$ ) and (c) DC bias for $L=900 \mathrm{~nm}$ and using $V_{A C}=10 \mathrm{~V}$. The size of the bubbles scales with the maximum of the DC to AC conversion efficiency, $\eta$, and is indicated for the corresponding generation band. 


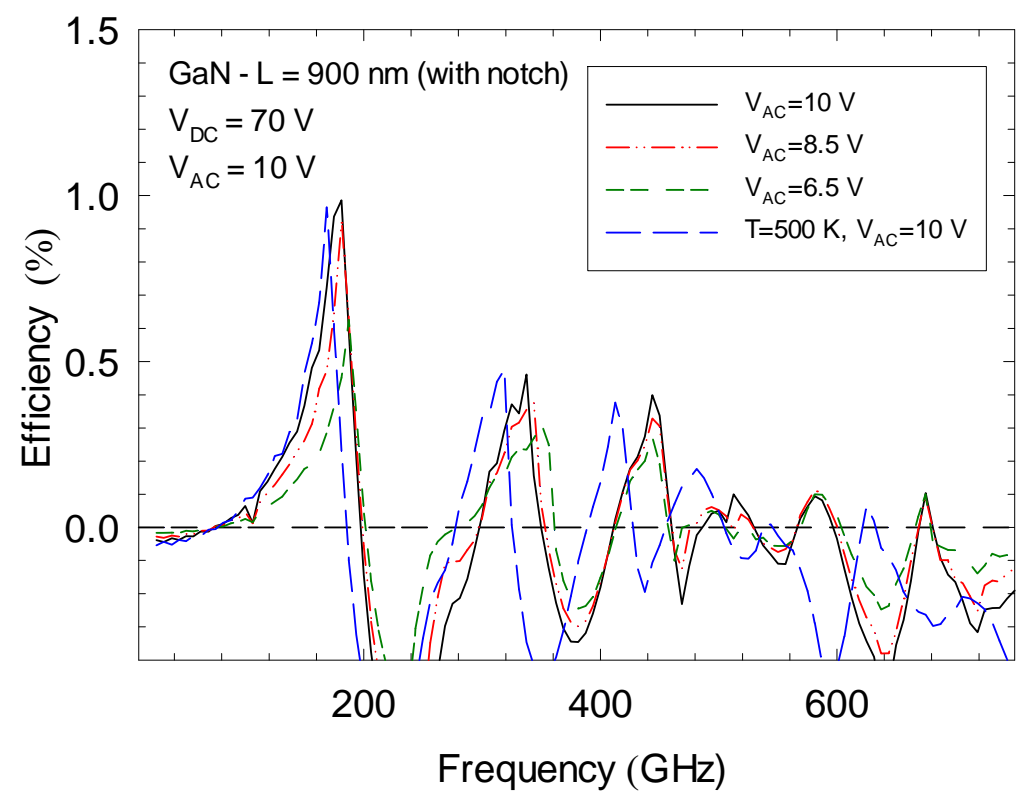

FIGURE 9. DC to AC conversion efficiency vs. frequency for a GaN-based diode with notch with $L=900$ $\mathrm{nm}$ and for the DS2 and with $V_{D C}=70 \mathrm{~V}$. The AC excitation used are $V_{A C}=10 \mathrm{~V}, 8.5 \mathrm{~V}$ and $6.5 \mathrm{~V}$. Also results $T=500 \mathrm{~K}$ are presents $\left(V_{A C}=10 \mathrm{~V}\right)$. 